\title{
TUMORES DEL OVARIO *
}

\author{
Doctor Carlos A. Goubert Laverde
}

Consideraciones sobre tumores del ovario

En el presente estudio se hace una revisión estadística minuciosa de los tumores ováricos hallados en el Hospital San Juan de Dios de Bogotá, de los años 1953 a 1960 inclusive y se trata a fondo el capítulo de los foliculomas.

\section{Material}

Para este trabajo que trata de ser uno de los más completos en cuanto a revisión numérica, no usamos los diversos ficheros del Hospital para la localización de los casos, sino que, personalmente buscamos uno a uno los protocolos quirúrgicos, de los años 1953 a 1960 inclusive, y luego sólo para comprobación de los casos hallados, hicimos un chequeo con los datos de dichos ficheros.

Se hallaron 24.887 protocolos quirúrgicos, de los que se estudiaron minuciosamente los correspondientes a las neoplasias ováricos y se buscaron y consultaron las historias clínicas del grupo de foliculomas; así como las placas histológicas, varias de las cuales fueron nuevamente revisados por el pa- tólogo doctor Egon Lichtenberger: se buscaron todas las macrofotografías de estos casos y practicamos personalmente nuevas fotomicrografías de las placas histológicas de algunos casos.

Clasificación de los tumores ováricos

Este es uno de los temas discutidos, debido a la naturaleza misma de estos blastomas, y muchos autores están de acuerdo, en que la clasificación dada por ellos, no es del todo satisfactoria, ya sea por muy elemental, o muy compleja, recargada con demasiadas subdivisiones originadas en detalles histológicos, embriológicos, etc., o por que no permite colocar en ella nuevos tipos encontrados, y debido a esto, existen gran cantidad de clasificaciones de los blastomas ováricos $(18,25,20,24)$ algunas de ellas muy conocidas por nosotros, como la de López Escobar (18).

Es necesario que nos detengamos a considerar la clasificación de la "American Medical Association", (3) cuya importancia radica en que permite ca-

* Trabajo presentado para ascender a Miembro de Número de la Sociedad Colombiana de Obstetricia y Ginecología. 
talogar las historias clínicas, para archivarlas adecuadamente según la etiología y topografía de la lesión, como a continuación se explica y preve además nuevas adiciones, sin que la clasificación sufra cambios fundamentales; también considera otros factores, como la presencia de esas lesiones con embarazo, etc.

Esta es la clasificación que se emplea en el archivo del Hospital San Juan de Dios de Bogotá, y en el Instituto de Protección Materno Infantil Concepción Villaveces de Acosta, y que permite un adecuado emp'eo del material científico allí recopilado, así como una rápida labor asistencial.

Clasificación de la A.M.A.

Enfermedades por disturbios circulatorios:

788-510.1. Necrosis de ovario por disturbio circulatorio.

Enfermedades por anomalías mecánicas o trastornos de la estática:

$788-637 *$. Torsión de pedículo ovárico.

Enfermedades por trastornos de metabolismo, crecimiento o nutrición:

788-796.7. Hemorragia de ovario por ruptura del folículo de Graaf.

788-795. Ovario quístico por falta de la involución.

7888-795. Cuerpo albicans quístico.

* Se citan estos numerales que no son de neoplasias para poder catalogar los casos de tumores ováricos complicados, con estos tipos de lesión sobreagregada,
7881-795. Quiste del folícu'o de Graaf.

7882-795. Cuerpo lúteo quístico.

7881-770. Ovarios poliquísticos, síndrome de Stein Leventhal.

\section{Tumores de ovario}

7887-8042. Arrenoblastoma de ovario.

788-8836. Tumor de Brenner.

788-8033. Cistadenoma de ovario.

788-8033 F. Cistadenocarcinoma de ovario.

788-881. Disgerminoma de ovario.

788-870 A. Fibroma de ovario.

788-8053. Tumor de células de la granu'osa de ovario.

788-8013. Tumor de Krukenberg de ovario.

788-8884. Mesonefroma de ovario.

788-8011. Cistadenoma seudomucoso de ovario.

788-8012. Cistadenocarcinoma pseudomucoso de ovario.

788-8021 F. Cistadenocarcinoma seroso papilar de ovario.

788-8021 B. Cistadenoma seroso papilar de ovario.

788-882.8. Teratoma quístico de ovario.

788-8052. Tecoma de ovario.

Tumores de ovario no incluídos en esta clasificación.

Enfermedades debido a causa desconocida o dudosa con reacción estructural manifiesta.

788-923. Calcificaciones de ovario.

788-952. Ooforitis crónica intersticial. 
788-911、 Degeneración quística de ovario.

788-959. Endometriosis de ovario.

7883-9 x 8. Quistes del epooforo.

7884-9 x 9. Quiste de paraoforo.

En esta clasificación se incluyen todas las enfermedades diagnosticables sin repetición; se emplean los términos ingleses aceptados, ocasionalmente se emplean términos latinos o griegos.

Se tienen en cuenta especialmente las denominaciones clínicas de las entidades así:

A. Localización topográfica.

B. Etiología.

Se designan con números de códigos separados por guión; los primeros tres dígitos describen la topografía, los tres siguientes al guión clasifican la etiología. $\mathrm{Su}$ combinación da la clasificación exacta, de la entidad.

Para dar mayor elasticidad a la clasificación se agregan dígitos decimales precedidos de un punto si el caso lo requiere para mayor exactitud.

$\mathrm{Si}$ el caso no se puede clasificar exactamente se pone la letra $Y$, ya sea para indicar la inexactitud en la topografía o en la etiología.

La letra $X$ en la clasificación topográfica indica órgano en sentido especial.

La letra $\mathrm{X}$ en la clasificación etiológica indica enfermedad de causa desconocida o incierta, que se manifiesta por reacción funcional.

Durante el embarazo cualquiera de las entidades clasificadas se diferenciaría cambiando un dígito por la letra $\mathrm{X}$.
$\mathrm{Si}$ a pesar de todas estas combinaciones posibles hay un caso que realmente no pueda ser clasificado, debe ser consultado con la A. M. A., para su inclusión en el presente índice.

\section{Frecuencia de los tumores ováricos}

Para establecer la frecuencia de los tumores ováricos se hace la relación del número de pacientes ingresados al servicio de Ginecología con el número de tumores hallados.

La frecuencia encontrada en el Hospital San Juan de Dios de Bogotá, de 1953 a 1960 inclusive es la que nos muestra el cuadro 1.

CUADRO 1

FRECUENCIA DE TUMORES

OVARICOS H. S. J. de D.

1953 a 1960

$N^{o}$ de pacientes $N^{o}$ de PorcentaAño hospitalizados tumores je anual

\begin{tabular}{rlrl}
\hline 1953 & $696 *$ & 138 & $20 \%$ \\
1954 & $695 *$ & 103 & $15 \%$ \\
1955 & 662 & 101 & $15 \%$ \\
1956 & 686 & 81 & $12 \%$ \\
1957 & 656 & 104 & $16 \%$ \\
1958 & 570 & 102 & $18 \%$ \\
1959 & 815 & 128 & $15 \%$ \\
1960 & 786 & 83 & $10 \%$ \\
\hline
\end{tabular}

La frecuencia promedio hallada en 8 años, fue de $15.1 \%$, pues en 5.566 pacientes hospitalizados en ginecología se hallaron 840 tumores ováricos.

* Cifra promedio, porque el número exacto no se encontró en el archivo. 
Otros autores dan las siguientes cifras (25):

$\begin{array}{ll}\text { Martin } & 1,4 \% \\ \text { Schmidlenchmer } & 1,44 \% \\ \text { Selye } & 2,8 \% \\ \text { Scanzoni } & 5,3 \% \\ \text { Velits } & 9,9 \%\end{array}$

Se puede explicar la disparidad de estos resultados porque en muchos servicios se hospitalizan casos que bien pudieran ser tratados como ambulatorios y otras veces las lesiones no neoplásicas se catalogan como parte de estas cifras.

Ahora veamos los tipos de tumores encontrados y la frecuencia de cada uno de ellos en el cuadro 2 .

\section{CUADRO 2}

FRECUENCIA DE LOS TUMORES DE OVARIO H. S. J. de D. 1953 a 1960

Nombre del tumor No de casos Porcentaje

Quistes policulares de retención

Quiste del cuerpo de retención

Ovario adenoquístico

Quistes endometriales

$260 \quad 30,95 \%$

Cistadenoma seroso

$43 \quad 5,12 \%$

Cistadenoma pseudomucoso

5

$0,59 \%$

Papiloma

Carcinoma primario quístico tipo cistadenocarcinoma

$91 \quad 10,83 \%$

Carcinoma primario sólido tipo adenocarcinoma

42

00 $5 \%$

Carcinoma metastásico o secundario

Fibromas

$15 \quad 1,79 \%$

Sarcomas

T. de Brenner

Tumor de células de la granulosa

Tecoma **

Arrenoblastoma

Disgerminoma

$\begin{array}{rl}9 & 1,07 \% \\ 11 & 1,31 \% \\ 1 & 0.12 \% \\ 1 & 0,12 \% \\ 6 & 0,71 \% \\ 6 & 0,71 \% \\ 2 & 0,23 \% \\ 4 & 0,48 \%\end{array}$

(Sigue)

** El caso 3434-59 fue primitivamente diagnosticado como tecoma, pero luego al revisar las placas surgieron dudas que no se pudieron aclarar satisfactoriamente, por lo cual no fue incluído en esta clasificación y se halla en el Departamento de Patología del Hospital San Juan de Dios.

El caso 1189-55 primitivamente diagnosticado como tecoma, fue revisado por dicho Departamento de Patología y definitivamente catalogado como arrenoblastoma. 
(Viene)

Nombre del tumor

Tumor de células lipoídicas

Quiste dermoide

$N^{O}$ de casos

0

119

0

4

Degeneración carcinomatosa de quistes dermoides

Tumores de tejidos neurales: neurofibroma, gangliocitoneuroma, etc.

Quistes ováricos no clasificados *
Total

Factor 0,1190477

* En casi todos los protocolos se explica que esta falta de clasificación es motivada por ausencia de epitelio en el interior del quiste para poder identificarlo; en algunos casos la clasificación fue imposible por los cambios sufridos en el tumor a consecuencia de la torsión del pedículo.

Por último se incluye en este grupo los casos diagnosticados como quistes simples de ovario.

Con fines comparativos mostramos la serie de Tumores de Ovario, revisada por Berstein (25), donde se analizan 1.101 casos hallados en el Hospital del Monte Sinai, y da las siguientes incidencias para ellos (cuadro 3 ):

\section{CUADRO 3}

FRECUENCIA DE LOS TUMORES DE OVARIO (14)

Quistes foliculares

$\%$

Quistes dermoides 36

Cistadenocarcinomas seroso papilar

Quistes del cuerpo lúteo

Carcinomas sólidos

Sarcomas

0,8

Teratomas malignos

0,7

Carcinoma secundario

0,5

Cacinoma escasomo en quiste dermoide

Seminoma

0,2

Carcinosarcoma

Así notamos que las cifras halladas por nosotros son bastante similares a las de Berstein, aun cuando difieren en algunos casos.

Es interesante analizar en forma pormenorizada algunos grupos de tumores:

14 Si tomamos el llamado grupo de "tu11 mores especiales" de la clasificación Cistadenomas pseudomucos a s papilares de G. López (18) que comprende los Endometriosis de ovario

Cistadenomas serosos papilares

Quistes tubo-ováricos

Fibromas siguientes blastomas: tumor de Bren4 ner, endometrioma, foliculoma, arre3 noblastoma, disgerminoma, y tumor de 2,6 células lipoídicas; veamos la relativa 2 gran frecuencia de estos tumores, pues entre 840 tumores ováricos hallamos 19 Cistadenocarcinomas pseudomucosos papilares 
similar a la dada por otros autores como Bourne A. (4), quien da $3 \%$.

Un dato interesante de obtener es el referente a tumores malignos. En el cuadro 4 sólo se hallan los tumores clásicamente tenidos por malignos, esto es:

\section{CUADRO 4}

\begin{tabular}{lcc}
\hline Tumor & $\begin{array}{c}N^{o} \text { de } \\
\text { casos }\end{array}$ & $\begin{array}{c}\text { Porcen- } \\
\text { taje }\end{array}$ \\
\hline Carcinoma primario & 40 & $4,77 \%$ \\
Carcinoma secundario & 9 & $1,07 \%$ \\
Sarcoma & 1 & $0,12 \%$ \\
Teratoma inmadiro & 0 & $0,0 \%$ \\
\hline
\end{tabular}

O sea un total de 50 tumores típicamente malignos, respecto de 840 tumores ováricos, que da un $5.9 \%$ de tumores malignos de ovario.

Estas cifras están en desacuerdo con las traídas por otros autores. Pero si consideramos todos los tumores ováricos malignos, incluyendo los tumores especiales malignos esto es: tumor de células de la granulosa, tecoma, arrenoblastoma, disgerminoma y tumor de células lipoidicas y descontamos los tumores no neoplásicos, tenemos que, sobre una serie total de 476 tumores ováricos, hay 68 malignos o sea en total $14,28 \%$ y es ya un porcentaje acorde con los citados por casi todos los autores como: Selye (25) que sobre una serie de 16.025 tumores ováricos, halla un $14,23 \%$ de tumores malignos, y Berstein (citado por Selye) quien en una de 1.101 tumores de ovario halla $17 \%$ de tumores malignos.
Como se recordará, el carcinoma de ovario es uno de los tumores genitales de la mujer de más difícil diagnóstico precoz debido a que son necesarios métodos de examen complicados como peritoneoscopia, etc. y por lo ambiguo de su sintomatología, la cual generalmente es ya tardía. Es uno de los tumores malignos bastante frecuentes, como nos lo muestra la estadística general de localización del cáncer en distintos órganos, elaborado por Cole W. colaboradores (8), en una obra sobre diseminación, prevención y terapia del cáncer, que vemos en el cuadro estadístico 5 donde el carcinoma ovárico actualmente ocupa el 7 lugar, con un 4,8\% .

\section{CUADRO 5}

Incidencia del carcinoma en la mujer

\begin{tabular}{lrr}
\hline Tipo de carcinoma & $\begin{array}{c}N^{o} \text { de } \\
\text { casos }\end{array}$ & $\begin{array}{c}\text { Porcen- } \\
\text { taje }\end{array}$ \\
\hline Seno & 52.000 & $21,8 \%$ \\
Cuello uterino & 25.000 & $10,5 \%$ \\
Piel & 24.000 & $10,1 \%$ \\
Intestino grueso & 22.000 & $9,2 \%$ \\
Estómago & 12.500 & $5,3 \%$ \\
Recto & 12.500 & $5,3 \%$ \\
Ovario & 11.500 & $4,8 \%$ \\
Cuerpo uterino & 8.000 & $3,4 \%$ \\
Aparato urinario & 7.000 & $2,9 \%$ \\
Linfomas & 6.000 & $2,5 \%$ \\
Hígado y vías biliares & 6.000 & $2,5 \%$ \\
Pulmón y bronquios & 5.000 & $2,1 \%$ \\
Leucemia y aleucemia & 5.000 & $2,1 \%$ \\
Páncreas & 4.000 & $1.7 \%$ \\
Cavida bucal y faringe & 4.000 & $1,7 \%$ \\
Cerebro y sistema & \\
nervioso & 3.000 & $1,3 \%$ \\
Otras localizaciones & 30.500 & $12,8 \%$ \\
\hline & & \\
\hline \multicolumn{2}{c}{ s. } \\
\hline
\end{tabular}


La presencia de lesiones quísticas no neoplásicas de ovario, ya sean solas o coexistiendo con otras lesiones de aparato genital, son muy frecuentes como nos lo indica el cuadro 6 .

CUADRO 6

\section{LESIONES NO NEOPLASICAS}

H. S. J. de D. 1953 - 1960

Tumores ováricos, 840 .

Lesiones no neoplásicas de ovario pero incluídas por muchos como "tumores ováricos" pues fácilmente se las equivoca macroscópicamente, 364 .

Porcentaje, 43,33\%.

Así se explican las diferencias aparentemente muy grandes de unos cuadros estadísticos con otros, como antes se analizó.

Bufe (25) considera que es difícil dar la incidencia de otras lesiones de una manera real porque en muchas ocasiones no se investigan; y dice, que si sobre un total de 100 ovarios escogidos al acaso provenientes de material de autopsias, por causas diversas se hace esta investigación, se hallan:

$\begin{array}{lr}\text { Folículos quísticos } & 77 \% \\ \text { Cuerpo lúteo quístico } & 9 \%\end{array}$

Para terminar esta larga serie de cuadros estadísticos generales, mencionaré el referente a la incidencia de los tumores ováricos según las edades, cuadro 7 .
CUADRO 7

FRECUENCIA DE TUMORES OVARICOS

H. S. J. de D. 1953 - 1960

\begin{tabular}{ccc} 
Edad & Porcentaje \\
\hline 10 a 19 & $7 \%$ \\
20 a 29 & $24 \%$ \\
30 a 39 & $30 \%$ \\
40 a & 49 & $25 \%$ \\
50 a 59 & $11 \%$ \\
60 a 69 & $2 \%$ \\
70 y más & $1 \%$ \\
\hline
\end{tabular}

Encontramos que los tumores de ovario predominan en la vida fértil de la mujer, pero que pueden hallarse en los extremos de la vida; además podemos observar que una mayor incidencia corresponde de los 30 a 39 años, dato en el cual están de acuerdo Berstein y Olshausen, citados por Selye (25).

Ahora a tratar a fondo el grupo de los foliculomas, comprendiendo tecomas y tumores de células de la granulosa, que según la opinión de muchos autores $(25,20,18)$ son aspectos de un mismo blastoma.

$\mathrm{He}$ escogido este grupo de tumores porque mientras yo era residente del Departamento de Ginecología del Hospita! San Juan de Dios de Bogotá, tuve ocasión de intervenir directamente en dos casos y seguir muy de cerca otros varios.

\section{Sinonimia}

Foliculoma-thelomas, tumor de Kahlden, tumor de células de la granulosa. 
Tecoma, tumor de células de la teca, fibroma thecocelular. Tumor de LöflerPriesel (25).

Mesenquimoma feminizante (13). Foliculoma maligno, carcinoma foliculoide de ovario, etc. (18).

\section{Incidencia}

Sobre una serie de 840 tumores ováricos (de protocolos quirúrgicos) en el Hospital San Juan de Dios, de los años 1953 a 1960 , hallamos 12 casos de foliculomas, lo que nos arroja una frecuencia global de $1,41 \%$. Hay 6 tumores de células de la granulosa y 6 tecomas, que da una relación de uno a otro del $50 \%$. Considerada aisladamente la frecuencia de los tumores de células de la granulosa respecto de los tumores ováricos malignos sólidos de los cuales hallamos 47 casos. Tenemos una incidencia de $12,7 \%$.

Faubert y Novak (20) opinan que es más raro el tecoma respecto del tumor de células de la granulosa; y para este (T. C. G.) le dan un $10 \%$ de los tumores ováricos malignos sólidos. López (18) da una incidencia global del 2 a $3 \%$. Sjösteds and Wahlen T. (26) en el sureste de Suecia, hallan una frecuencia de 1 x 100.000 para mujeres entre 19 y 53 años, y de 1 x 54.000 entre las de 50 a 64 años.

Si consideramos que en la serie total revisada por nosotros, de 24,887 protocolos anatomopatológicos de piezas quirúrgicas de toda clase, se hallaron 19 especímenes de tumores funcionantes ováricos, o sea un $0,076 \%$ y lo comparamos con una serie presentada por el doctor Litchtenberger E. (17) de 5.401 protocolos anatomopatológicos que recogen prácticamente todos los casos registrados en el antiguo Laboratorio de Pato'ogía del Hospital San Juan de Dios desde la fundación de dicho laboratorio, y vemos que sólo hay 1 caso de tumor funcionante de ovario, denominado tumor de células luteínicas, o sea $0,018 \%$.

Si cotejamos los dos porcentajes hallados vemos que el de la primera serie es 4 veces mayor; esto se explica porque antes se diagnosticaban muchos tumores funcionantes erróneamente como fibromas, sarcomas, etc., de ovario.

Por esta razón no establecemos en la otra serie cifras numéricas.

\section{Edad}

En la serie estudiada hay 4 casos entre $\operatorname{los} 30$ y 39 años, 4 casos de 40 a 49 años, 2 casos de 50 a 59 años y 2 casos de 60 a 69 años, lo cual muestra el gran predominio de estos tumores en el final de la vida fértil de la mujer y en la menopausia.

\section{Paridad}

Estos tumores son una causa de esterilidad de tipo endocrino por su potencial estrongénico con producción de ciclos anovulatorios y se halla demostrado claramente en uno de nuestros casos, en el cual después de una esterilidad secundaria de 2 años y 9 meses, se extirpó 1 foliculoma, y 4 meses después de salir la enferma del hospital, regresa a control y se halla en gestación normal de 3 meses. 
El que no hubiera mayor número de embarazos posteriores a la intervención, se puede explicar entre otras razones porque la edad de este grupo de pacientes oscila en gran parte entre los 30 y 49 años; y porque muchos tumores no son endocrinamente activos, y al extirparlos no habrá cambios hormonales en dichas pacientes.

\section{CUADRO 8}

$P A R I D A D$

H. S. J. de D. - 1953 - 1960

\begin{tabular}{|c|c|c|}
\hline $\begin{array}{l}N^{o} \text { de } \\
\text { hijos }\end{array}$ & $\begin{array}{c}N^{o} \text { de } \\
\text { casos }\end{array}$ & $\begin{array}{l}\text { Tiempo entre el } \\
\text { último parto y la } \\
\text { aparición del tumor }\end{array}$ \\
\hline 0 & 4 & - \\
\hline 1 & - & - \\
\hline 2 & 1 & No sabe \\
\hline 3 & 2 & 2 a. $9 \mathrm{~m}$. \\
\hline 4 & 1 & No sabe \\
\hline 5 & - & - \\
\hline 6 & 1 & No sabe \\
\hline 7 & - & - \\
\hline 8 & 1 & No sabe \\
\hline Sin dato & 2 & - \\
\hline
\end{tabular}

\section{Localización}

H. K. Farrar y R. Bryan (8) revisan el debatido tema del predominio de blastomas en el ovario izquierdo, y sólo hallan un ligero predominio en éste sobre el derecho. En nuestra corta serie de 12 casos no podemos hablar de porcentaje, sino de número de casos, como lo muestra el cuadro 9, que está de acuerdo con las estadísticas anteriores.
Israel (12) opina que el tumor de células de la granulosa es 4 veces más frecuentemente bilateral que el tecoma, pero en nuestra corta serie sólo hallamos 2 casos de tumores bilaterales y ambos eran tecomas. Recuérdese que se trata de una serie de sólo 12 casos, y ésto nos podría explicar la diferencia.

\section{CUADRO 9}

LOCALIZACION DEL TUMOR

H. S. J. de D. 1953 - 1960

\begin{tabular}{lccc}
\hline \multicolumn{5}{c}{$\begin{array}{c}\text { Ovario } \\
\text { Tipo }\end{array}$} & $\begin{array}{c}\text { Ovario } \\
\text { derecho }\end{array}$ & izquierdo & Bilateral \\
\hline Tecoma & 1 & 3 & 2 \\
$\begin{array}{l}\text { Tumor de cé- } \\
\text { lulas de la } \\
\text { granulosa }\end{array}$ & 2 & 4 & 0 \\
\hline
\end{tabular}

La frecuencia de tumores ováricos sólidos bilaterales, debe hacernos pensar casi siempre en malignidad.

\section{Sintomatología}

Clasificaremos en dos grupos los síntomas de estos tumores: uno relacionado con la presencia de tumor ovárico, de relativo grado de malignidad, y ésto nos explicará la masa en fosa ilíaca, la ascitis, etc.; y otro grupo referente a la producción hormonal estrogénica, que en opinión de muchos como Mackey y colaboradores (citado por 18) es debida a las células tecales.

Recuérdese que a pesar de ser uno de los tumores llamado funcionante, un porcentaje grande de ellos no son hor- 
monalmente activos y por lo tanto no se presentarán los síntomas del último grupo.

En el cuadro 10 se muestran en orden decreciente los síntomas hallados en esta serie, que son casi todos los síntomas clásicos pero debemos hacer resaltar que el motivo de consulta fue generalmente la masa palpable abdominal y/o el do!or abdominal.

\section{CUADRO 10}

CARACTERISTICAS DE LOS FOLICULOMAS

H. S. J. de DIOS - 1963 - 1960

\begin{tabular}{lc}
\hline Síntomas & No de casos \\
Masa palpable & 12 \\
Dolor hipogástrico & 7 \\
Amenorrea en período fértil & 5 \\
Esterilidad primaria & 4 \\
Metrorragias post-menopáusicas & 3 \\
Ascitis & 3 \\
Disuria & 3 \\
Menopausia sin síntomas subjetivos & 2 \\
Retención urinaria & 2 \\
Incontinente urinaria por rebosamiento & 1 \\
Cuadro abdominal agudo & 1 \\
Circulación colateral & 1 \\
Anemia y mal estado general & 1 \\
Disnea & 1 \\
Tos & 1 \\
Edema de miembros inferiores & 1 \\
Hidrotórax derecho (a los R. X.) & 1 \\
Hidrotórax izquierdo (a los R. X.) & 1 \\
Esterilidad secundaria & 1 \\
Asintomáticos & 0 \\
\hline
\end{tabular}

Si consideramos que algunos casos como el correspondiente a la historia clínica 64.482 y 185.850 son el cuadro de un tumor maligno con metástasis, vemos que la consulta fue tardía para algunas enfermas.
Al reunir los casos de amenorrea, esterilidad primaria o secundaria y los de metrorragias, se agrupa en otro gran bloque de motivos de consulta, que las mujeres llaman familiarmente "desarreglos menstruales" y que como 
en los casos presentes obedecen a causas serias. Así pues, no debemos juzgar a la ligera de síntomas que a veces parecen motivados por causas banales, que si se hubieran estudiado a fondo y más tempranamente es probable nos hubieran hecho sospechar un blastoma ovárico funcionante; antes de que se presentaran otros como gran tumor, etc.

Es oportuno recordar la necesidad de valorar todos los datos en pacientes con ginecopatías, ya que en nuestra serie ningún caso fue diagnosticado como tumor funcionante y sólo se mencionó vagamente esta posibilidad en 1 caso.

Cuando clínicamente hallamos hidrotórax y masa abdominal baja, debemos pensar antes que en un síndrome de Meigs en lesiones malignas de ovario pues el fibroma de ovario tiene una frecuencia de $1,31 \%$ contra un $14,28 \%$ de lesiones ováricas malignas, capaces de provocar un hidrotórax, según nuestras estadísticas y aún mayores para autores como Berstein (cit. 14).

Ninguno de los casos de esta serie fue asintomático y por lo tanto de hallazgo ocasional, sino que todas consultaron por ginecopatías.

No se anotan problemas pre-puberales y todas las pacientes presentan una pubertad que oscila entre los 12 y los 15 años, clínicamente normal.

Se incluyen además en este cuadro signos clínicos menores cuyo único objeto es darnos una cabal idea del estado de los pacientes.

En el estudio pre-operatorio de las mismas, se deben pedir algunos exáme- nes paraclínicos, sin los cuales corremos el riesgo de hacer un mal diagnóstico pre-operatorio, y así valorando equivocadamente el caso, comentamos serios errores.

CUADRO 11

EXAMENES PARACLINICOS

$$
\text { H. S. J. de D. } 1953 \text { - } 1960
$$

Casos

Exámenes de rutina preoperatorio 11

Curetaje uterino 3

Biopsia de cérvix 3

R. de Galli Mainnini 3

Citología en líquido acítico para buscar células tumorales 3

Chequeo cardiovascular 2

Biopsia por congelación 1

Citología vaginal 1

Cistoscopia 1

Sin exámenes 1

En el servicio de ginecología se considera como exámenes de rutina preoperatorio: parcial de orina, azohemia, glicemia, cuadro hemático, eritrosedimentación, serología, grupo sanguíneo y $\mathrm{R} \mathrm{H}$, fotofluorografía de tórax y opcional coproanálisis. Además se pedirán los exámenes que se consideren oportunos según el caso.

A los enfermos con cardiopatías manifiestas o mayores de 50 años con leves signos o síntomas cardiovasculares se pide concepto cardiovascular al Departamento de cardiología en donde la enferma es estudiada a fondo para valorar el riesgo quirúrgico a que se la va a someter.

En el cuadro anterior vemos que sólo una paciente no tuvo exámenes de 
laboratorio preoperatorios, y está justificado ante el diagnóstico y adecuada valoración clínica evidentes, y la dificultad de laboratorio a altas horas de la noche.

El que sólo se hubieran practicado 3 curetajes uterinos con 3 biopsias de cervix nos recuerda de hacer hincapié en la necesidad de estos exámenes como rutina para pacientes en quienes se sospechan neoplasias malignas porque dan multitud de datos; y si se considera la facilidad de practicarlos en el consultorio debemos aceptar que respecto a este punto las pacientes fueron mal estudiadas.

Otro examen de laboratorio muy fácil de practicar es la citología vaginal para estudio funcional, y si bien es cierto que los datos obtenidos en este procedimiento son casi los mismos que los de la biopsia endometrial respecto a niveles hormonales; en las circunstancias que esta no sea posible o aconsejable debe practicarse la citología vaginal, y anotamos que el único método práctico de apreciar el nivel estrogénico es la biopsia endometrial y la citología vaginal, pues técnicas para dosificar los metabolitos urinarios como estroma estradiol 17 - B y estriol son largos y laboriosos como lo muestra la técnica de Brown (22).

La citología del líquido ascítico en busca de células tumorales, la biopsia operatoria por congelación y la cistoscopia son procedimientos que se emplearon con toda propiedad en estas pacientes cuando fueron requeridos.

Se practicaron 3 biopsias intraoperatorias para tener un dato más al tomar la decisión quirúrgica definitiva, conducta muy buena si se tiene en cuenta que la biopsia era lo único que faltaba para terminar el estudio completo de estas pacientes.

Antes de entrar a analizar los cuadros 12 y 13, hacemos notar que un mismo caso tuvo varias hipótesis diagnósticas, y todas ellas se hallan aquí consignadas por lo cual el número total de diagnósticos es superior al de casos reales.

CUADRO 12

DIAGNOSTICO PREOPERATORIO H. S. J. de D. 1953 - 1960

\begin{tabular}{lc}
\hline & Casos \\
\hline Carcinoma ovárico & 5 \\
Fibromatosis uterina & 2 \\
Tumor ovárico con partes sóli- & \\
das y quísticas & 2 \\
Tumor ovárico sólido & 2 \\
Sarcoma uterino & 1 \\
Adenocarcinoma endometrial & 1 \\
Tumor ovárico "Funcionante" ? & 1 \\
Embarazo (para descartar) & 1 \\
Fibroma ovárico & 1 \\
Abdomen agudo por quiste ová- & \\
rico torcido & 1 \\
\hline
\end{tabular}

$$
\text { CUADRO } 13
$$

DIAGNOSTICO PREOPERATORIO H. S. J. de D. 1953 - 1960 PATOLOGIA ASOCIADA

\begin{tabular}{lc}
\hline & Casos \\
\hline Cervicitis crónica & 5 \\
Cistorrectocele & 3 \\
Hiperplasia endometrial & 2 \\
Quiste Gl. de Bartholin & 2 \\
Cistocele & 1 \\
Histerocele 111 & 1 \\
Ascitis & 3 \\
\hline
\end{tabular}


En el cuadro 12 en el que se agrupan los diagnósticos preoperatorios vemos que muchos casos en los cuales se hallaron masas anexiales poco móviles, irregulares, difíciles, de delimitar como carcinomas ováricos sin mayores consideraciones sobre otras lesiones, malignos sí, pero no directamente carcinomas de ovario que como sabemos no tienen el mismo pronóstico.

Los casos diagnosticados como tumor sólido, o tumor con partes quísticas y sólidas, etc., son buenos diagnósticos semiológicos que no prejuzgan de la naturaleza misma de los blastomas, y en los tres casos se comprobó la exactitud de estos diagnósticos.

El único abdomen agudo fue ocasionado por torsión del pedículo del tumor y lo consideramos como un accidente poco frecuente en tumores que tienden a formar adherencias.

Los demás diagnósticos fueron errados.

En el cuadro 13 se agrupan los diagnósticos preoperatorios de patología asociada, de los que solamente fueron comprobados histopatológicamente las cervicitis, pues en los demás por la naturaleza misma de la intervención no hubo lugar a practicar dichos estudios, y los diagnósticos eran obvios.

$\mathrm{Si}$ parangonamos los diagnósticos preoperatorios con los hallazgos histológicos del cuadro 14 tenemos que hacer varias anotaciones: No fue diagnosticado clínicamente ningún caso como foliculoma, ni siquiera como hipótesis remota.

Hubo una confirmación absoluta del estado de los 5 cervix estudiados y catalogados clínicamente como servicitis crónica.

No encontramos ningún caso de hiperplasia endometrial o adenocarcinoma de endometrio que es una asociación citada frecuentemente con los tumores feminizantes por muchos autores como Dockerty y colaboradores (citado por 18), Greener (10), West and Rawson (28). Israel (14) explica que una estimulación estrogénica prolongada produce hiperplasia endometrial y a menudo alto índice de mitosis; y D Ward (7) completa esta aseveración apuntando que además influye la baja de progesterona en estas pacientes para dar la mencionada asociación.

Fueron errados los diagnósticos de sarcoma uterino, de miomatosis uterina como lesión principal, y no se sospecharon las metastasis a peritoneo.

CUADRO 14

DIAGNOSTICOS ANATOMOPATOLOGICOS

H. S. J. de D. 1953 - 1960

Lesión principal Casos

Tecomas 6

Tumor de células de la granulosa 6 Total 12

Patología asociada

Cervicitis crónica 5

Miomatosis uterino 2

Adenocarcinoma de trompa 1

Pólipos endometria!es 1

Salpingitis crónica bilateral 1

Metástasis a peritoneo 1

Torsión e infarto hemorrágico

de anexo 
CUADRO 15

TIPO DE OPERACION

H. S. J. de D. 1953 - 1960

\begin{tabular}{lc}
\hline Intervención & Casos \\
\hline Incisión longitudinal & 1 \\
Incisión transversal & 1 \\
$\begin{array}{l}\text { Salpingooforectomía bilateral } \\
\text { (SOB) }\end{array}$ & 6 \\
Salpingooforectomía unilateral & 3 \\
(SOU) & 3 \\
Histerectomía total y SOB & 2 \\
Miomectomía & 1 \\
Biopsia de fondo uterino y vejiga & 1 \\
Laparotomía exploradora & 1 \\
\hline
\end{tabular}

La biopsia de fondo uterino y vejiga a que se refiere el cuadro 15 fueron practicadas a cielo abierto duran. te la laparotomía tomando fragmentos cuneiformes de los tejidos sospechosos de éstos órganos.

$\mathrm{Al}$ analizar las incisiones vemos que hay una marcada tendencia al uso de las longitudinales aun cuando autores de la categoría de Alvarez - Bravo (2), Greenhill (11) y Rodríguez López (22), anotan las enormes ventajas de las transversales sobre las primeras en abdomen. En publicación anterior (9) hemos analizado el pro y contra de esta vía de abordaje abdominal y los resultados obtenidos, siendo muy favorables para las incisiones transversales.

Las líneas generales de conducta en cuanto a cirugía radical están bastante claras teóricamente, pero en ocasiones el problema surge al tomar la decisión quirúrgica en el caso particular. Recordemos que siguiendo estas ideas de hacer la cirugía lo menos mutilante po- sible en la mujer joven, se permiten nuevas gestaciones normales después de extirpar un folículoma como en el caso citado por nosotros.

La conducta radical propuesta por algunos autores como Alberti (1) Randal y Telinde (27) entre otros es hoy prácticamente rechazada.

El que sólo se practicaran 3 histerectomías totales con $\mathrm{SOB}$, y sí 9 anexectomías; algunas de ellas en pacientes post-menopáusicas, podemos considerarlo acorde con las ideas expresadas.

El caso en el que se proponían realizar histerectomía total con SOB y sólo se efectuó la SOB y toma de biopsia de fondo uterino y vejiga, ante el hallazgo a la laparotomía es aceptable, si se piensa que el resultado de aquellas biopsias orientaría a la conducta futura respecto a irradiaciones, etc., y que de momento se evitarían graves lesiones especialmente del tipo de fístulas; y como clínicamente parecía tratarse de un estado inoperable pues las posibles metástasis ya estarían fuera de alcance de la cirugía, sería causar lesiones innecesarias.

Las complicaciones, cuadro 16 , son las corrientes citadas por casi todos los autores, exceptuando el caso de inoperabilidad y el de peritonitis fibrino-purulenta postoperatoria. En los trabajos hechos en el Servicio de Ginecología del Hospital San Juan de Dios. Goubert C. (9) en el referente a insiciones abdominales curvas en cirugía ginecológica y obstétrica, y Rodríguez A. (23) en el suyo sobre histerectomías también traen datos similares sobre complicaciones. 
CUADRO 16

COMPLICACIONES

H. S. J. de D. 1953 - 1960

Quirúrgicas

Casos

Técnicamente inoperable 1

Post-operatorias

Fiebre (por encima de $38^{\circ} \mathrm{C}$. o que excede de esta cifra desde el segundo día postoperatorio persistiendo por dos días o más) 4

Retardo en la cicatrización

Meteorismo marcado

Retención urinaria cuando no se aplicó sonda vesical postoperatoria a permanencia

Insuficiencia cardíaca

Peritonitis y muerte cuenta que autores como Sjöstedtsand Wahlen (26) dan a veces supervivencias de 15 a 20 años para los $2 / 3$ de los pacientes. Kottmeir (15) opina que es necesario separar las estadísticas de los carcinomas ováricos tipos y otros tumores ováricos malignos, pues estos últimos son de mucho mejor pronóstico.

Los escandinavos Kullander S., Källen B. y colaboradores (16) logran novísimos datos obtenidos por cultivo de tumores ováricos humanos y su comportamiento bajo la influencia hormonal, diferentes en parte a los obtenidos por Raven y colaboradores (21) antes. Pero las conclusiones terapéuticas y pronósticos que se pueden obtener de dichos estudios no están bien definidas aún y todavía, estas investigaciones están en el terreno de la ciencia pura.

A la única paciente que sabemos falleció, se le practicó autopsia y el resultado es el siguiente:

Historia clínica No 64-482,

Consideramos como complicación quirúrgica el caso de aquella paciente en la cual se planteó tal o cual técnica operatoria y solamente después al realizar la laparotomía, ante el cuadro de invasión carcinomatosa se decidió no practicar ninguna intervención. $\mathrm{Al}$ hablar de "pronóstico" nos referiremos a le peritonitis.

\section{Pronóstico}

Se trata de una serie reciente para juzgar de la mortalidad, si se tiene en
A. P. No 830-55.

Fallecimiento: 17-V-55.

Resumen de lesiones:

"Histerectomía total con SOB, por tecoma, de ovario izquierdo, cervicitis crónica, pólipos endometriales, (Prot. No 830/55). Peritonitis fibrino-purulenta (800 cc.), congestión y edema pulmonar; hidrotórax bilateral (izq. 300 cc., der., 50 cc.); colapso basalpulmonar; degeneración grasosa de hí- 
gado; proctitis ulcerosa aguda (bacteriana); hemorragia vesical (traumática) ; bocio coloide nodular, con adenomas foliculares (cuerpo tiroides 130 gramos).

\section{Resumen:}

Paciente de 60 años de edad sometida a histerectomía total con SOB, que muere 5 días después de intervenida con peritonitis fibrino-purulenta generalizada".

Así a pesar de que una paciente de esta serie murió, poco después de operada (5 días) no podemos juzgar de la malignidad en esta serie nuestra; porque todos son casos muy recientes y esta muerte no es imputable directamente al tumor en sentido de malignidad, pero sí problema de mortalidad por histerectomía total con SOB.

Por último en el cuadro 17 se mencionan el número del Protocolo de $\mathrm{Pa}-$ tología y el número de historia clínica para enfocar mejor los casos, se analiza el tiempo transcurrido entre la aparición de los primeros síntomas y la primera consulta, lo cual nos da un promedio de 383,34 días, o sea aproximadamente un año, tiempo que es muy corto para marcar el comienzo de la enfermedad, si se piensa que muchos eran ya blastomas de grandes o me- dianas dimensiones, y vemos una vez más, la necesidad de campañas bien orientadas en la lucha contra el cáncer.

El tiempo de estudio en consulta externa fue en promedio de 25 días, que no es muy alto si se tiene en cuenta que varios casos tuvieron hospitalizaciones previas en medicina interna, etc., y luego fueron remitidos de allí a ginecología.

El tiempo postoperatorio 13,5 días, es una cifra promedio de las que trae el bolecín de estadística mensual del Hospital, referente a ginecología en el período al cual pertenecen estos casos.

El total de días de hospitalización es bastente largo pero si lo descomponemos en sus detalles, encontramos que es a expensas del tiempo preoperatorio o donde se alarga inútilmente dicho período.

Presentamos a continuación una corta serie de macro-fotografías y fotomicrografías de los foliculomas de esta serie realizadas por el autor inclusive en la parte de laboratorio.

El material gráfico presentado no es de la calidad deseada, porque los originales (propiedad del Hospital) de los cuales se partió, son deficientes, pero los únicos disponibles. 


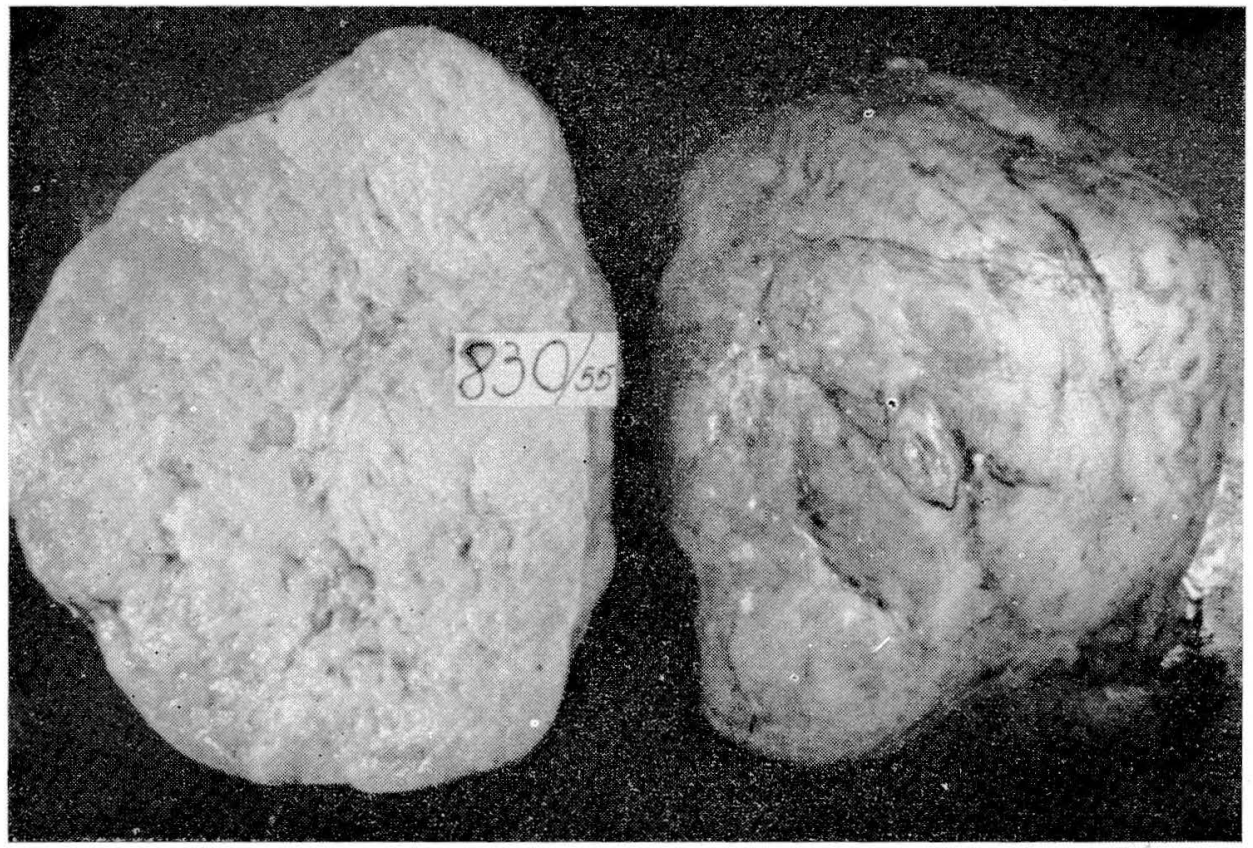

Figura 1. Tecoma de ovario. Case: 830-55. Tumor seccionado mostrando su cara convexa y plano de corte. Tiene aspecto típico de un tumor ovárico sólido de consistencia elástica; que recuerda vagamente el cuadro de los leiomiomas uterinos.

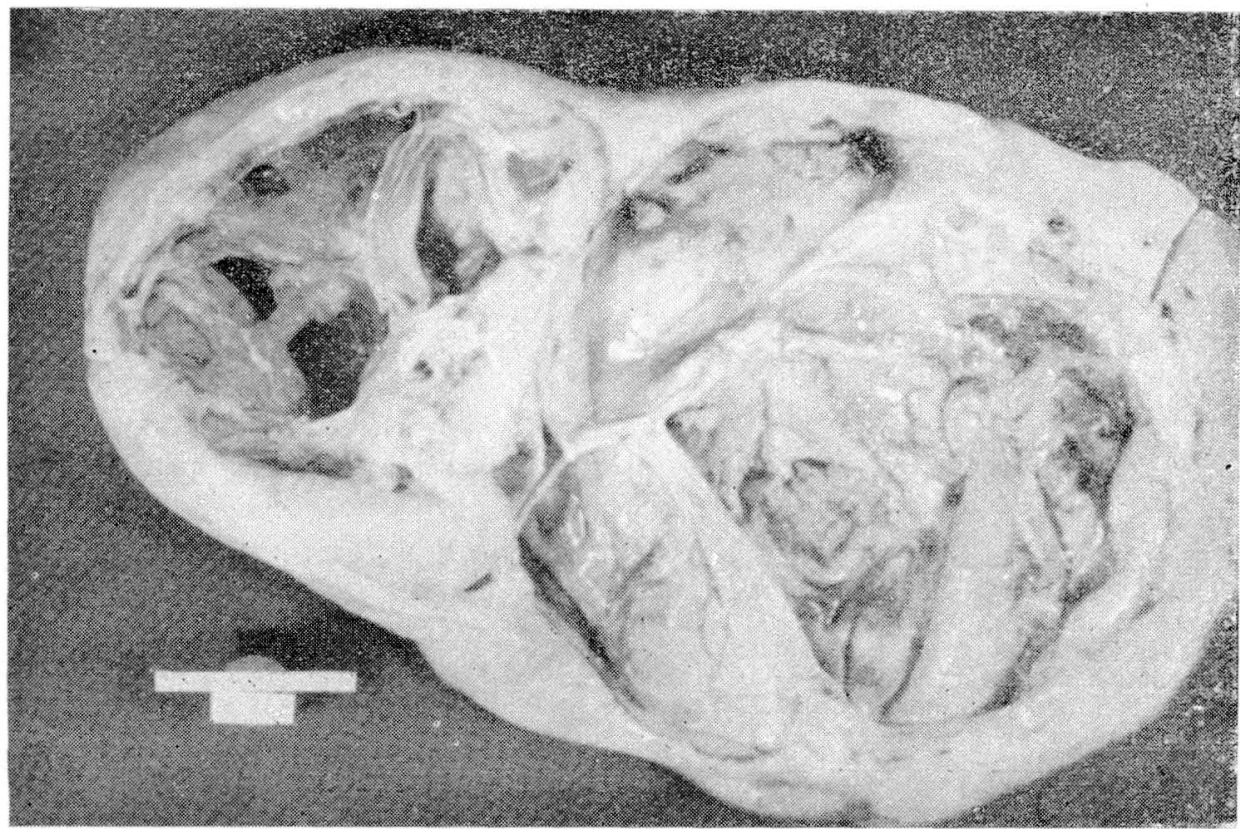

Figura 2. Tumor de células de la granulosa. Caso: 566-56. La pieza nos muestra un tumor ovárico con partes sólidas y cavidades que antes estuvieran llenas de líquido formado de quistes irregulares. 


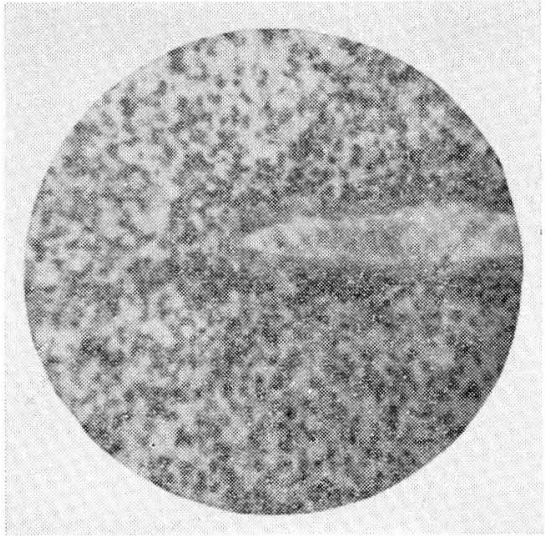

Figura 3. Tumor de células de la granulosa. Caso: 499-59. Hay gran cantidad de estroma y un vaso sanguíneo en sección oblicua. Vemos gran cantidad de células epiteliales de citoplasma claro, de límites mal definidos. El cuadro no es típico y vagamente recuerda el de un disgerminoma.

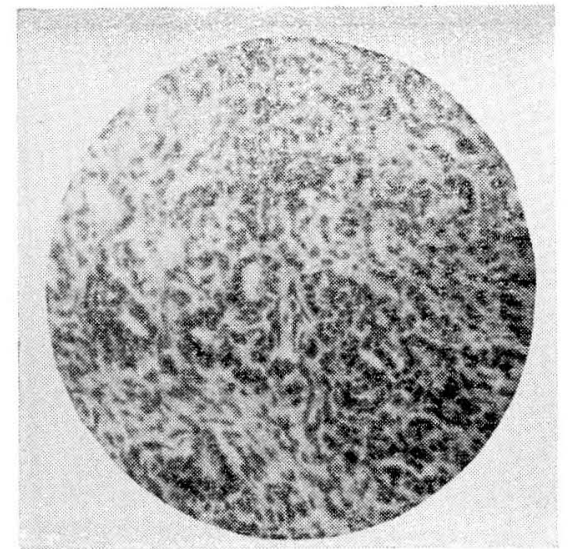

Figura 5. Tumor de células de la granulosa. Caso: 427-59. Estroma constituído por células fusiformes y cilíndricas. Observamos túbulos constituídos por células epiteliales. El conjunto es el cuadro típico de un tumor de células de la granulosa de ovario.

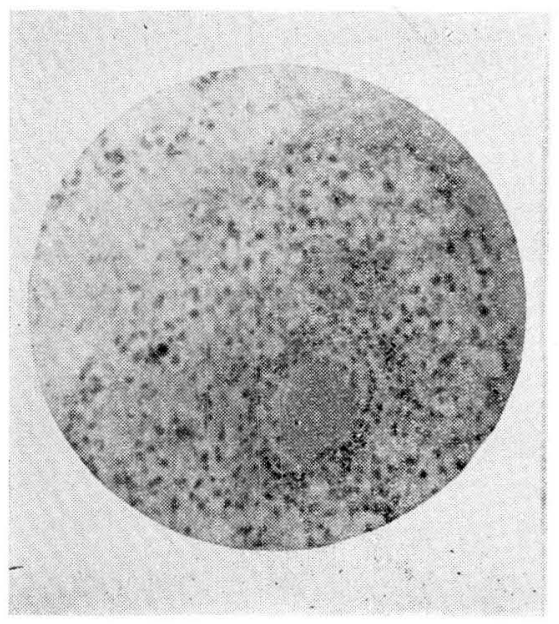

Figura 4. Tumor de células de la granulosa. Caso: 499-59. Encontramos un vaso sanguíneo en sección transversal células epiteliales $y$ estroma infiltrado por linfocitos.

\section{Conclusiones}

1. Se deben codificar los diagnósticos según normas internacionales para poder archivar y consultar adecuada y fácilmente el material c'ínico.

2. Al analizar resultados estadísticos debemos tener en cuenta las cifras globales y los factores que intervinieron para así poder comparar nuestros datos con los de otros autores y saber si la concordancia o diferencia son reales o ficticias; porque sin este análisis existe el peligro de cometer graves errores.

3. Es necesaria la revisión de las placas histológicas muy antiguas, porque muchos blastomas ováricos se hallan mal clasificados. 
4. Hay que analizar a fondo cada uno de los síntomas y signos de la paciente y luego coordinarlos para formular diagnósticos genéricos, semiológicos, y si es posible precisar luego más, para evitar el gran porcentaje de errores diagnóstico que hemos mostrado.

5. Es conveniente practicar más exámenes paraclínicos especiales según cada caso, pues a pesar de que las cifras encontradas no son malas, vemos que se hubieran podido estudiar mejor algunas de las pacientes de nuestra serie.

6. Investigar en series mayores la asociación de hiperplasia o adenocarcinoma endometrial y foliculomas, pues la presente es muy corta para poder juzgar sobre este punto.

7. Se recalcan las ventajas del uso de la incisión transversa en cirugía tocoginecológica.

8. Practicar una cirugía lo más funcional posible sobre todo en mujeres jóvenes pero evitar conservar órganos enfermos en pacientes preclimatéricos o aún mayores.

9. Necesidad de un buen archivo fotográfico.

10. Mejor estudio de las pacientes en consulta externa para así obtener una estancia preoperatoria más corta.

11. Hacer una profilaxia efectiva del cáncer, comenzando por llegar al gran núcleo de mujeres y explicar la necesidad de dichas campañas, de las cuales ellas serán las directamente beneficiadas.
12. Tratar de obtener un mayor control posterior de las pacientes que como todos sabemos es muy difícil. Quizás explicándoles la necesidad y conveniencia para ellas en primer lugar, de este control; y agilizando extremadamente para estas enfermas los trámites administrativos, es posible obtengamos mejor índice de controles y así nos daremos cuenta cabal de la evolución de nuestras pacientes a lo largo del tiempo.

\section{Sumario}

1. Se presenta un estudio estadístico sobre todo el material de protocolos quirúrgicos del Hospital San Juan de Dios de Bogotá, entre los años 1953 - 1960; 24.887 en total, y de allí se estudian estadísticamente en detalle todos los blastomas ováricos hallados: 840 en total.

2. Sólo se insiste en un aspecto de la clasificación de tumores ováricos; el de la A.M.A. que permite codificar los diagnósticos una vez clasificados, y así ordenar lógicamente la estadística hospitalaria de acuerdo a normas internacionales para fácil consulta y comparación con la de muchos hospitales que usan este sistema.

3. Los principales temas analizados en nuestra estadística global sobre tumores ováricos son: frecuencia por años y total; variedades halladas e incidencia; por centaje de tumores malignos; y por último promedio de edades para todos los blastomas.

4. Se desarrolla un completo estudio estadístico de un grupo de blasto- 
mas ováricos: los foliculomas con 12 casos. Se estudian: sinonimia, incidencia, edad, paridad, localización, sintomatología, exámenes para-cínicos, diagnósticos preoperatorios, diagnósticos anatomopatológicos, tipo de operación, complicaciones, pronóstico y datos cronológicos.

5. Se presenta una serie de Macro y foto-microfotografías de los foliculomas.

6. Conclusiones.

\section{BIBLIOGRAFIA}

1. AlBerti y PIOVANO: Bolet. Soc. Obst. y Gin. de Buenos Aires, XXXIV. No 488. Agosto 1955.

2. AlVAREZ BRAVO: Incisión transversa. Gin. y Obst. de México. Sept. Oct. pág 297 y sig.

3. American Medical Association Standard Nomenclature of Diseases and Operations. Mc Graw Hill. Book Co. N. Y. 5 ed. 1961.

4. BOURNE A. W. and Williams L. H.: Recent Advances in Obstetrics and Ginecology. Págs. 283, 284, 305, 307, 315. The Blakiston Company, Philadelphia. Ed. 1945.

5. CARDOnA P.: Dosificación de estrógenos en orina. Rev. Col. de Obst. y Gin. Vol. XI. № 1. Ene. Feb. 1960. Pág. 50 y sig.

6. COLEW. Mac DONALD G., ROBERTS S., SOUTHWICK H.: Disemination of cancer - Prevention and therapy. Appleton - Century Grafts Inc. Ed. New York 1961. Pág. 22.

7. DE WARD. Acta Endochinológica, 29: 279, 1958.

8. FARRAR H. K., Jr. and BRYAN R.: Year Book of Patology and Clinical Pathology, 1960- 1961. Ed. Year Book Medical. Publishers. Chicago.

9. GOUBERT C.: Incisiones transversas curvas en cirugía ginecológica y obstétrica. Rev. Col. de Obst. y Gin. Vol. X. Nov. 1959. Pág. 289 y sig.

10. GREENER Jr. J. W.: Am. J. Obst. \& Gynec. 74: 31-40. July 1957.

11. GREEN HILL J. P.: Transverse incision in pelvic surgery. Year Book of Obs. and Gin. 1955-1956. Pág. 309 y sig.

12. ISRAEL S. L.: Surg. Gynec \& Obst. 105: 166 176. August. 1957.

13. ISRAEL S. L.: Recent Advances in Obstetrics and Ginecology. Pág. 496. The Blakinton Company. Philadelphia. Ed. 1945.

14. ISRAEL S. L.: Geriatrics 14: 488 - 495. Aug. 1959.

15. KOTTMEIER H. L.: Fourt National Cancer Conference Proceeding 1960. J. B. Lippincott Company, Phyladelphia. 1961. 
16. GULLANDRES and KALLEN B.: Acta Obstétrica y Ginecológica Scandinávica. XL fasc. 4: 391-393-401.

17. LICHTENBERGER E.: Estadística de 5.401 casos estudiados en el Laboratorio de Patología del H. S. J. de Dios de Bogotá; tesis de grado act. 1947. Biblioteca del Departamento de Patología del H. S. J. de Dios.

18. LOPEZ E. G.: Medicina y cirugía. Vol. XV: Nọ 8. 1951.

19. Mac CALL: ML.: Year Book of Obstetrics \& Gynecolofy 1958-1959. Págs.: 473, 474, 475. The Year Book Publisher, Chicago.

20. NOVAK and NOVAK: Tratado de Ginecología. M. V. Fresneda. Habana. Ed. 1958. Pág. 456 y sig., 520-532.

21. RAVEN R. W.: Cancer. Págs. 172, 180, 181. Vol. 1. Butterworth \& Co. Publishers Ltd. London 1957.

22. RODRIGUEZ LOPEZ: Rápida incisión de Pfannestiel. Year Book of Obs. \& Gin. 1948. Pág. 443 y 44.

23. RODRIGUEZ SOTO A.: Histerectomía Rev. Col. de Obs. y Gin. Vol XII. N. 4. Jul. Agos. 1961. Pág. 295 y sig.

25. SELYE H.: Enciclopedia of Endocrinoly. Section IV Ovary. Vol VII. Richardson, Bond and Wright. Montreal 1946.

26. SJOOSTEDTSAND and WAHLER T.: Acta Obstétrica y Ginecológica Sdandinávica. XL Suplement. 6: 17-24-25. 1961.

27. TE LINDER R. W.: Gincelogía operatoria. Ed. José Bernardes. 1956. Pág. 677.

28. West. Ch. y RAWSON R.: Progreso de la ginecología. Vol. 11. Ed. Científico Médica, Barcelona. 1958. Pág. 331. 